\section{A NOVEL INTERPRETATION OF TRANSDUCER POWER GAIN BY OPTICAL ANALOGY}

\author{
Hsuan-Yu Pan, ${ }^{1}$ Hsing-Yuan Tu, ${ }^{1}$ Shey-Shi Lu, ${ }^{1}$ and C. C. Meng ${ }^{2}$ \\ ${ }^{1}$ Department of Electrical Engineering \\ National Taiwan University \\ Taipei, Taiwan, R.O.C. \\ ${ }^{2}$ Department of Electrical Engineering \\ Chung-Hsing University \\ Taichung, Taiwan, R.O.C
}

\section{Received 4 May 2001}

ABSTRACT: A novel interpretation of transducer power gain $\left(G_{T}\right)$ is presented by optical analogy. By employing scattering parameters of the input / output matching network and the transistor, we have developed a simple method to derive $G_{T}$. In addition to simplicity, our theory also has the advantage of giving the physical meaning for each term of the formula of $G_{T}$. (c) 2001 John Wiley \& Sons, Inc. Microwave Opt Technol Lett 31: 124-126, 2001.

Key words: transducer power gain; scattering parameters; transistor

\section{INTRODUCTION}

It is well known that the transducer power gain $\left(G_{T}\right)$ is one of the most important figures of merit in the design of microwave transistor amplifiers. Several methods [1-4] have been proposed to derive the transducer power gain. However, most of them rely heavily on mathematical calculations rather than simple physical reasoning, and therefore it is difficult to give a physical meaning to each term in the formula of the transducer power gain $G_{T}$. In view of this, we have developed a simple and intuitive way to derive $G_{T}$ by means of optical analogy. In the derivation, the concept of incident and reflected waves is employed to simplify the circuit analysis, and the physical insight into each term in $G_{T}$ is naturally obtained. Our theory also provides a useful transformation between the different methodologies of optics and microwave circuit design.

\section{THEORY}

The block diagram of a microwave transistor amplifier is shown in Figure 1(a), where $V_{S}$ is the voltage source, $Z_{O}$ is $50 \Omega$, and $\Gamma_{S}$ and $\Gamma_{L}$ are the source and load reflection coefficients in a $Z_{O}$ system. $P_{A V S}$ and $P_{L}$ represent the available power of the signal source and the power delivered to the load $Z_{O}$, respectively. The input and output matching networks are assumed to be passive and lossless.

In order to obtain the transducer power gain, i.e., $P_{L} / P_{A V S}$, by optical analogy, we first replace components in the microwave circuit by optical components. The resultant optical system is shown in Figure 1(b), where the signal source in the amplifier has been replaced by a light source with power of $P_{A V S}$, the input matching network by a lossless partially transmitting mirror with a reflection coefficient of $\Gamma_{S}$ (at plane 2) looking toward the source, the output matching network by a lossless partially transmitting mirror with a reflection coefficient of $\Gamma_{L}$ (at plane 5) looking toward the load, and finally, the transistor by an optical gain medium with the scattering parameters of the transistor. Note that $Z_{O}$ s, because of their nonreflecting property in a $Z_{O}$ system, were replaced by empty space in this optical system. Also note that $\Gamma_{S}$, in fact, is the $S_{22}$ of the input mirror and $\Gamma_{L}$ is the $S_{11}$ of the output mirror. Because any linear network that contains no controlled sources will be reciprocal [5], the $S$-matrix of the input/output matching network will be symmetric [6]. Further, owing to the lossless property of the input/output matching network, the $S$-matrix is also unitary [6]. It is proven that the magnitudes of $S_{11}$ and $S_{22}$ of a symmetric network will be equal [7], and hence the magnitude of the reflection coefficient $\Gamma_{S}^{\prime}$ (i.e., $S_{11}$ ) of the input mirror at plane 1 looking toward the load is equal to that of $\Gamma_{S}$, namely,

$$
\left|\Gamma_{S}^{\prime}\right|=\left|\Gamma_{S}\right|
$$

Equation (1) is the key point of our proposed theory. The $S$-matrix of the input mirror can be easily derived from (1) due to its lossless and unitary properties $[6,7]$ as follows:

$$
[S]_{\text {input }}=\left[\begin{array}{cc}
\Gamma_{S}^{\prime} & \sqrt{1-\left|\Gamma_{S}\right|^{2}} e^{j \phi 1} \\
\sqrt{1-\left|\Gamma_{S}\right|^{2}} e^{j \phi 1} & \Gamma_{S}
\end{array}\right]
$$

where $\phi 1$ is an arbitrary phase angle. Similarly, the $S$-matrix of the output mirror can be written as

$$
[S]_{\text {output }}=\left[\begin{array}{cc}
\Gamma_{L} & \sqrt{1-\left|\Gamma_{L}\right|^{2}} e^{j \phi 2} \\
\sqrt{1-\left|\Gamma_{L}\right|^{2}} e^{j \phi 2} & \Gamma_{L}^{\prime}
\end{array}\right]
$$

where $\phi 2$ is another arbitrary phase angle.

When the light source with power $P_{A V S}$ is incident from the left on the input mirror as shown in Figure 1(b), it is partially reflected and partially transmitted. According to basic electromagnetic theory [8], the transmitted power is

$$
\left|a_{1}\right|^{2}=P_{A V S} \times\left(1-\left|\Gamma_{S}^{\prime}\right|\right)^{2}=P_{A V S} \times\left(1-\left|\Gamma_{S}\right|\right)^{2}
$$

where $a_{1}$ is the normalized amplitude of the transmitted light. However, this transmitted light continues to the optical gain medium. Here, the portion $S_{11} \cdot a_{1}$ is reflected, and the portion $S_{21} \cdot a_{1}$ is transmitted. The reflected part is returned to the input mirror and then reflected: $\Gamma_{S} \cdot S_{11} \cdot a_{1}$. This amplitude, reflected from the input mirror, goes through the same process as the initially transmitted light $a_{1}$. Each repetition of the process amounts to multiplication by $\Gamma_{S} \cdot S_{11}$. Therefore, the total wave leaving the input mirror and incident on the gain medium (see Fig. 2) is

$$
a=a_{1}\left(1+\Gamma_{S} \cdot S_{11}+\left(\Gamma_{S} \cdot S_{11}\right)^{2}+\cdots\right)=\frac{a_{1}}{1-\Gamma_{S} \cdot S_{11}}
$$

where $a$ is the normalized amplitude of the total wave. Since the power of a wave is the square of its amplitude, the actual 


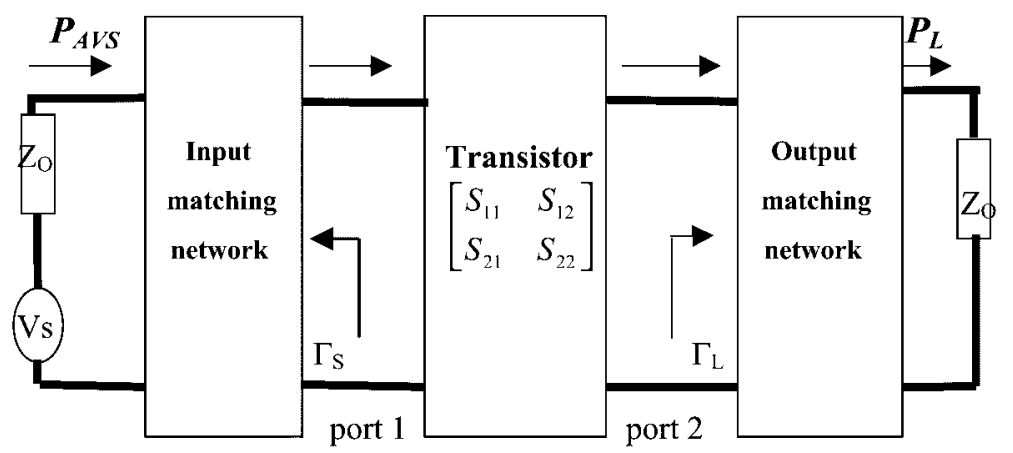

(a)

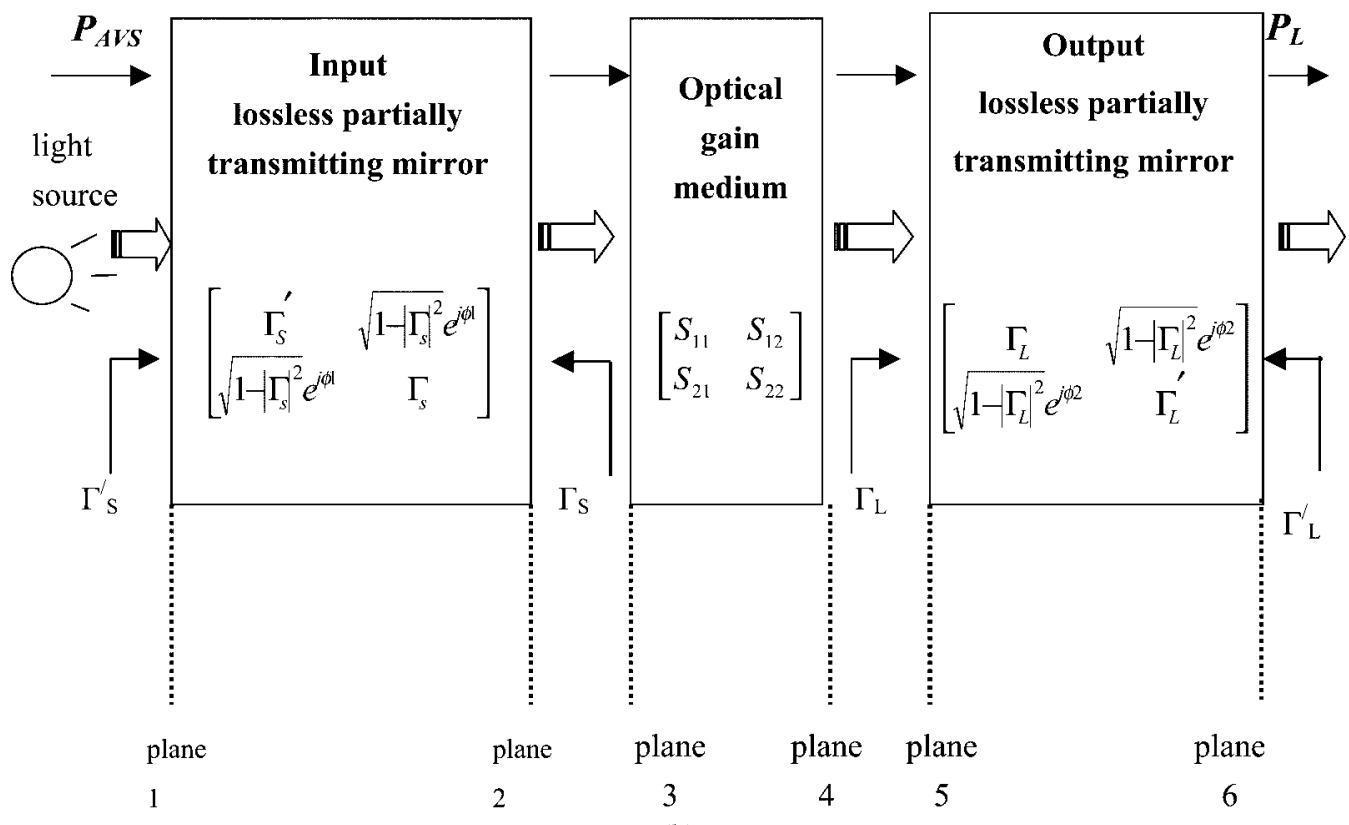

(b)

Figure 1 (a) Block diagram of a microwave amplifier. (b) Optical analogy of the microwave amplifier circuit in (a)

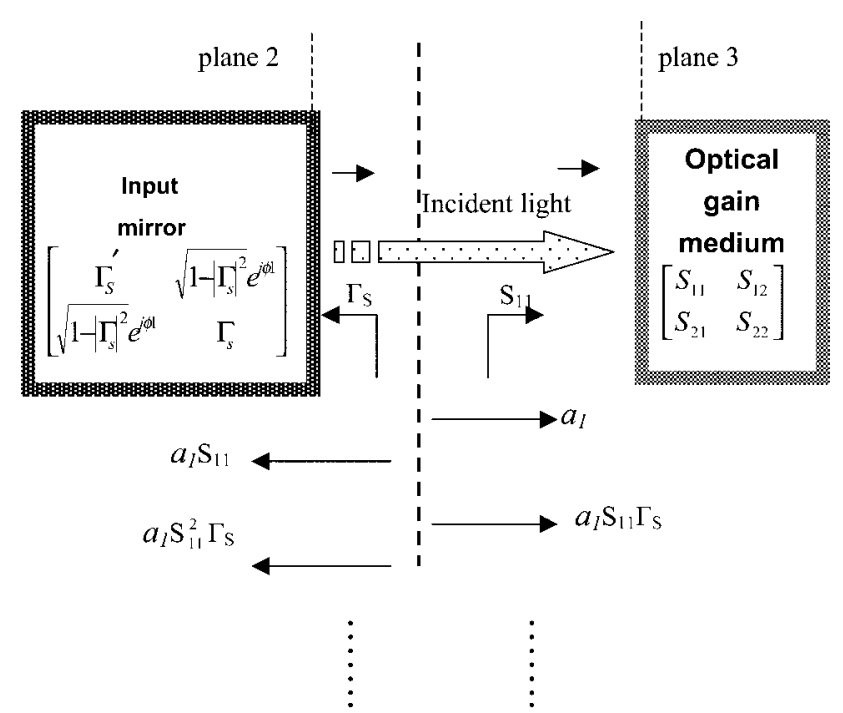

Figure 2 Schematic diagram showing the wave reflections between the transistor and the input mirror power incident into the gain medium is

$$
|a|^{2}=\frac{\left|a_{1}\right|^{2}}{\left|1-\Gamma_{S} \cdot S_{11}\right|^{2}}=P_{A V S} \frac{1-\left|\Gamma_{S}\right|^{2}}{\left|1-\Gamma_{S} \cdot S_{11}\right|^{2}} .
$$

From the definition of $S_{21}$, the power coming out of the gain medium can be easily written as

$$
\left|a_{2}\right|^{2}=\left|a \cdot S_{21}\right|^{2}=P_{A V S} \frac{1-\left|\Gamma_{S}\right|^{2}}{\left|1-\Gamma_{S} \cdot S_{11}\right|^{2}}\left|S_{21}\right|^{2}
$$

where $a_{2}$ is the normalized amplitude of the light just coming out of the gain medium. When $a_{2}$ arrives at the output mirror, a portion $\Gamma_{L} \cdot a_{2}$ is reflected. The reflected part is returned to the gain medium, where a portion $S_{22} \cdot \Gamma_{L} \cdot a_{2}$ is reflected and a portion $S_{12} \cdot \Gamma_{L} \cdot a_{2}$ is transmitted. The transmitted part continues to the input mirror, and is reflected: $\Gamma_{S} \cdot S_{12} \cdot \Gamma_{L} \cdot a_{2}$. This reflected wave experiences multiple reflections between the input mirror and the gain medium, and finally, a total wave incident on the gain medium is $\Gamma_{S} \cdot S_{12}$. $\Gamma_{L} \cdot a_{2} /\left(1-\Gamma_{S} \cdot S_{11}\right)$, which is then transmitted through the 
gain medium with an amplitude of $S_{21} \cdot \Gamma_{S} \cdot S_{12} \cdot \Gamma_{L} \cdot a_{2} /(1-$ $\left.\Gamma_{S} \cdot S_{11}\right)$. This amplitude has to be added to the initial reflected wave $S_{22} \cdot \Gamma_{L} \cdot a_{2}$, and their sum becomes

$$
\begin{aligned}
S_{22} \cdot & \Gamma_{L} \cdot a_{2}+\frac{S_{21} \cdot \Gamma_{S} \cdot S_{12} \cdot \Gamma_{L} \cdot a_{2}}{1-\Gamma_{S} \cdot S_{11}} \\
& =\left(S_{22}+\frac{S_{21} \cdot \Gamma_{S} \cdot S_{12} \cdot \Gamma_{L} \cdot a_{2}}{1-\Gamma_{S} \cdot S_{11}}\right) \cdot \Gamma_{L} \cdot a_{2} \\
& =\Gamma_{\text {out }} \cdot \Gamma_{L} \cdot a_{2}
\end{aligned}
$$

where $\Gamma_{\text {out }}$ is defined as $S_{22}+S_{21} \cdot \Gamma_{S} \cdot S_{12} /\left(1-\Gamma_{S} \cdot S_{11}\right)$. This summed reflected wave in (8) goes through the same process as the initially transmitted light $a_{2}$. Clearly, each repetition of the process amounts to multiplication by $\Gamma_{\text {out }}$. $\Gamma_{L}$. Therefore, the total wave leaving the gain medium and incident on the output mirror (see Fig. 3) is

$$
a_{3}=a_{2}\left(1+\Gamma_{\text {out }} \cdot \Gamma_{L}+\left(\Gamma_{\text {out }} \cdot \Gamma_{L}\right)^{2}+\cdots\right)=\frac{a_{2}}{1-\Gamma_{\text {out }} \cdot \Gamma_{L}}
$$

and its associated power is

$$
\begin{aligned}
\left|a_{3}\right|^{2} & =\left|\frac{a_{2}}{1-\Gamma_{\text {out }} \cdot \Gamma_{L}}\right|^{2} \\
& =P_{A V S} \frac{1-\left|\Gamma_{S}\right|^{2}}{\left|1-\Gamma_{S} \cdot S_{11}\right|^{2}}\left|S_{21}\right|^{2} \frac{1}{\left|1-\Gamma_{\text {out }} \cdot \Gamma_{L}\right|^{2}}
\end{aligned}
$$

where $a_{3}$ is the normalized amplitude of the total wave incident on the output mirror. Since the reflection coefficient of the output mirror is $\Gamma_{L}$, it is clear that the power $P_{L}$ coming out from the output mirror is

$$
\begin{aligned}
P_{L} & =\left|a_{3}\right|^{2}\left(1-\left|\Gamma_{L}\right|^{2}\right) \\
& =P_{A V S} \frac{1-\left|\Gamma_{S}\right|^{2}}{\left|1-\Gamma_{S} \cdot S_{11}\right|^{2}}\left|S_{21}\right|^{2} \frac{1-\left|\Gamma_{L}\right|^{2}}{\left|1-\Gamma_{\text {out }} \cdot \Gamma_{L}\right|^{2}} .
\end{aligned}
$$

$P_{L}$ corresponds to the power delivered to the load in the microwave amplifier, and hence from the above equation

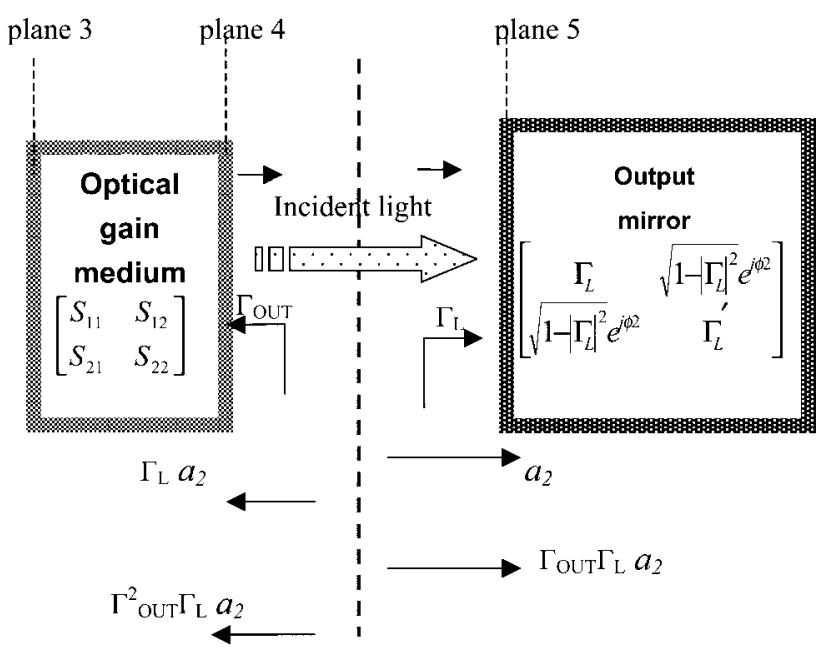

Figure 3 Schematic diagram showing the wave reflections between the transistor and the output mirror, as well as those between the transistor and the input mirror
(11), the transducer power $G_{T}=P_{L} / P_{A V S}$ can be found to be

$$
G_{T}=\frac{1-\left|\Gamma_{S}\right|^{2}}{\left|1-S_{11} \Gamma_{S}\right|^{2}}\left|S_{21}\right|^{2} \frac{1-\left|\Gamma_{L}\right|^{2}}{\left|1-\Gamma_{\text {out }} \Gamma_{L}\right|^{2}} .
$$

\section{CONCLUSION}

A novel interpretation of the formula of transducer power gain was presented. The derivation was based on the scattering parameters of the input/output matching network and the transistor and optical system associated with them. It was found that $1-\left|\Gamma_{S}\right|^{2}$ represents the fraction that the signal power transmits through the input matching network, and $1 /\left|1-\Gamma_{S} \cdot S_{11}\right|^{2}$ accounts for the multiple reflections of the transmitted wave between the input matching network and the transistor. $\left|S_{21}\right|^{2}$ is the power gain of the transistor. $1 /\left|1-\Gamma_{L} \cdot \Gamma_{\text {out }}\right|^{2}$ takes into account the multiple reflections between the transistor and the output matching network, as well as those between the input matching network and the transistor.

\section{REFERENCES}

1. G. Gonzalez, Microwave transistor amplifiers, Prentice-Hall, Englewood Cliffs, NJ, 1997, 2nd ed., pp. 175-194.

2. G.D. Vendelin, Microwave circuit design using linear and nonlinear techniques, Wiley, New York, 1990, pp. 54-64.

3. R. Ludwig, RF circuit design, Prentice-Hall, Englewood Cliffs, NJ, 2000, pp. 466-470.

4. D.M. Pozar, Microwave engineering, Wiley, New York, 1998, pp. 606-609.

5. A.B. Carlson, Circuits, Brooks/Cole, Thomson Learning, Inc., 2000, pp. 650-651.

6. D.M. Pozar, Microwave engineering, Wiley, New York, 1998, pp. 199-201.

7. G.D. Vendelin, Microwave circuit design using linear and nonlinear techniques, Wiley, New York, 1990, p. 70, problem 1.15.

8. D.K. Cheng, Field and wave, electromagnetics, Addison-Wesley, Reading, MA, 1989, 2nd ed., pp. 397-401.

(c) 2001 John Wiley \& Sons, Inc.

\section{IMPROVEMENT OF MODULATION/ SWITCHING PERFORMANCES BY USING A TWO-SECTION SEMICONDUCTOR OPTICAL AMPLIFIER}

\author{
Hong Wu Li, ${ }^{1}$ Thierry Rampone, ${ }^{1}$ and Mikael Guegan ${ }^{1}$ \\ ${ }^{1}$ Laboratoire RESO \\ Ecole Nationale d'Ingénieurs de Brest \\ Technopôle Brest-Iroise, BP 30815 \\ 29608 Brest Cedex, France
}

\section{Received 7 May 2001}

ABSTRACT: By optimizing the carrier distribution along the longitudinal axis of a two-section semiconductor optical amplifier (SOA), we have improved the modulation / switching performances (modulation index and efficiency, switching contrast) with respect to those of a single-section component. The modulation efficiency has been raised by up to 137\%. (c) 2001 John Wiley \& Sons, Inc. Microwave Opt Technol Lett 31: 126-129, 2001.

Key words: semiconductor optical amplifiers; modulation; switching; two-section $S O A$ 